\title{
Mali: Les exciseuses persistent malgré les supplications
}

Frontiers in Reproductive Health

Follow this and additional works at: https://knowledgecommons.popcouncil.org/departments_sbsr-rh

Part of the International Public Health Commons, Public Health Education and Promotion Commons, and the Women's Health Commons How does access to this work benefit you? Let us know!

\section{Recommended Citation}

"Mali: Les exciseuses persistent malgré les supplications," FRONTIERES Résumés de Recherche Opérationnelle. Washington, DC: Population Council, 2000. 
Mali

Excision

$N^{\circ} 2$

\section{Les exciseuses persistent malgré les supplications}

Les stratégies mises en ouvre pour convaincre les exciseuses

traditionnelles d'abandonner la pratique de l'excision sont inefficaces. Elles devraient en effet se focaliser sur l'élimination de la demande pour cette pratique et non sur la réduction de l'offre de service.

\section{Contexte}

Environ 94 pourcent des femmes maliennes âgées de 15 à 49 ans ont subi l'excision. Dans ce pays, l'excision est associée à de sérieuses complications gynécologiques et obstétriques (ASDAP, DFSC, 1998).

En 1998, le Centre National de Recherche Scientifique et Technologique du Ministère malien des Enseignements secondaire, supérieur, et de la recherche scientifique a mené une étude visant à évaluer les stratégies destinées à éradiquer l'excision. Cette évaluation est partie du travail de trois ONG locales actives dans la ville de Bamako et dans cinq autres régions : Association Malienne de Suivi et d'Orientation des Pratiques Traditionnelles/AMSOPT, Association pour le Progrès et la Défense des Droits des Femmes/APDF, et Association de Soutien au Développement des Activités de Population/ASDAP.

\section{Les exciseuses continuent leur} pratique. La plupart d'entre elles restent convaincues que l'excision n'est pas une pratique néfaste pour les femmes.

L'objectif de ces stratégies était de convaincre les exciseuses à abandonner la pratique et à devenir les relais de la sensibilisation. Ces exciseuses sont pour la plupart de la caste des forgerons et sont issus des familles reconnues par la communauté comme étant exciseuses.
Les trois ONG ont employé des travailleurs sociaux pour sensibiliser les exciseuses et la communauté sur les effets néfastes de l'excision sur la santé des femmes. Deux d'entre elles ont notamment développé des activités alternatives génératrices de revenus pour ces exciseuses alors que la troisième $a$ cherché à leur faire accepter l'arrêt de la pratique. En plus des leaders des trois ONG, 10 agents de santé et 41 exciseuses ont été interviewés; de même 45 groupes de discussion ont été organisés avec 380 membres de la communauté.

\section{Résultats}

- Presque toutes les familles pratiquent l'excision. Sur un échantillon nonreprésentatif de 126 femmes âgées de moins de 40 ans, 91 pourcent ont affirmé avoir excisé leurs filles. Néanmoins, la pratique pourrait décliner puisque ce sont 98 pourcent des 134 femmes âgées de plus de 40 ans qui ont déclaré avoir excisé leurs filles.

- Les principaux responsables de la prise de décision concernant l'excision sont des chefs de famille, des leaders religieux, des chefs de villages et des grands-mères. La communauté considère l'excision comme un moyen de perpétuer les traditions, de s'acquitter des obligations religieuses, de contrôler la sexualité féminine, et de préparer les filles au mariage. 
- Des membres de la communauté et le personnel local des ONGs ont rapporté que les exciseuses continuent la pratique en dépit de leur engagement à abandonner l'excision. Les exciseuses qui ont réellement abandonné la pratique avancent deux principales raisons : (1) leur âge très avancé, la baisse de leur capacité visuelle ou leur remplacement par leurs filles; et (2) la promesse d'activités alternatives génératrices de revenus. Cependant la grande majorité de ces exciseuses restent convaincues que l'excision n'est pas nuisible aux femmes.

\section{Les programmes d'éradication de} l'excision doivent atteindre diverses cibles parmi lesquelles les femmes, les hommes, les jeunes, les leaders d'opinion, les chefs religieux, et les accoucheuses traditionnelles.

- Les stratégies de reconversion ont été inefficaces parce que :

$\nLeftarrow$ Les parents continuent de recourir aux exciseuses quand le besoin se présente. Ils trouvent également du personnel se santé prêt à exciser leurs filles.

$\leftrightarrow$ Le statut social des exciseuses ne leur permet pas de prendre des décisions unilatérales lorsqu'il s'agit de mettre fin à l'excision.

$\leftrightarrow$ Les exciseuses jouissent d'une certaine reconnaissance par la communauté. Ainsi la rétribution qu'elles reçoivent pour leur travail n'est pas leur seule source de motivation.

\section{Des hommes parlent de l'excision :}

« Le monde change. C'est pourquoi nous pouvons maintenant parler d'excision avec vous. Avant, personne ne serait venu discuter de ce sujet.»

«Peut-être que nos petits-fils ne feront pas d'excision. Mais dans tous les cas son abandon n'interviendra pas tant que nous sommes en vie.»

Participants à des discussions de groupe

\section{Implications pour les programmes}

- Les programmes doivent se focaliser sur la réduction de la demande des familles pour l'excision au lieu de chercher à réduire l'offre des services par les exciseuses.

- Les ONG doivent développer de vastes campagnes d'éducation de base dans la communauté pour promouvoir une discussion sur l'excision et encourager les dirigeants locaux à se prononcer contre la pratique. Les programmes d'éradication de l'excision doivent atteindre diverses cibles parmi lesquelles les femmes, les hommes, les jeunes, les leaders d'opinion, les chefs religieux, et les accoucheuses traditionnelles.

- Les recherches doivent être axées sur des stratégies d'intervention efficaces s'appuyant sur la santé de la reproduction et les droits de l'Homme, rejeter les arguments avancés par les partisans de l'excision, et en documenter les leçons apprises.

Ministère des Enseignements Secondaire, Supérieur et de la Recherche Scientifique, Centre National de la Recherche Scientifique et Technologique et Population Council. «Evaluation de la Reconversion des Exciseuses pour l'Eradication des Mutilations Génitales Féminines au Mali ». Bamako, Mali. 1998. Pour de plus amples informations contacter : Population Council, 128 Sotrac Mermoz, BP : 21027, Dakar, Sénégal. Tel. : 221-824-19-93 ; Fax : 221-824-19-98 ; Email : pcdakar@pcdakar.org. 